\title{
Projet de révision de la statistique médicale de la FMH
}

Esther Kraft,

Martina Hersperger

Par ordre alphabétique: ASMAC, Bureau BASS, CMPR, College-M, NewIndex, OBSAN, OFS, OFSP, SSPP, Université de Berne (excusée: Société suisse de pédiatrie).

Correspondance:

FMH Secrétariat général

Département DDQ

Elfenstrasse 18

CH-3000 Berne 15

ddq@fmh.ch

\section{Situation initiale}

Depuis un certain temps, il est incontesté que la statistique médicale de la FMH, sous sa forme actuelle [1], ne peut répondre que de manière partiellement satisfaisante aux questions qui se posent en matière de démographie médicale. Le Comité central a par conséquent décidé ce printemps de lancer un projet de «Révision de la statistique médicale FMH» et a chargé le domaine Démographie, données et qualité (DDQ) de le mener à bien. L'état actuel du projet est décrit dans les lignes qui suivent.

\section{Tâches, teneur et limites de la statistique} médicale révisée de la FMH

Les médecins et leur profil personnel et professionnel constituent le point central de la statistique médicale de la FMH. Celle-ci doit donc offrir des informations sur l'ensemble des médecins suisses, informations portant sur leurs caractéristiques sociodémographiques, l'état de leur formation prégraduée, postgraduée et continue et leur situation professionnelle actuelle. L'activité professionnelle devrait être recensée de façon différenciée:

- lieu: où se situe le lieu de travail du médecin? (p.ex. au cabinet médical, à l'hôpital);

- contenu: quelle est la nature de son travail? (p.ex. médecin de premier recours, spécialiste);

- ampleur: combien travaille-t-il? (p.ex. activité à temps complet, à temps partiel).

La statistique médicale de la FMH ne donne pas, ni ne donnera à l'avenir, d'autres renseignements concernant, par exemple, l'infrastructure du cabinet ou les prestations qui y sont fournies. Avec les informations qu'elle livre sur la démographie médicale, la statistique de la FMH apporte une contribution spécifique aux recherches sur la fourniture de soins en Suisse, tout en complétant et facilitant les efforts similaires déployés par d'autres institutions dans ce domaine.

\section{But du projet et procédure}

Le projet a pour objectif de fournir à la FMH des bases de données de bonne qualité et bien acceptées, qui permettent d'effectuer, aujourd'hui comme à l'avenir, une évaluation fiable des questions de démographie médicale. A cet effet, il faut, premièrement, utiliser dans la statistique médicale de la FMH des définitions et des indicateurs adaptés aux questions qui se posent actuellement et, deuxièmement, relever les données de la manière la plus exacte possible tout en les actualisant régulièrement. Le projet prévoit deux phases: durant l'année en cours, les variables et les indicateurs qui fondent la statistique médicale, ainsi que leur définition, devront être réexaminés et corrigés si nécessaire (p.ex. en ce qui concerne l'activité en cabinet médical, les médecins de premier recours, les taux de travail à temps partiel). En 2008, une procédure de relevé et d'actualisation périodique des données sera développée et mise en application. On a beau choisir et définir les variables de la façon la plus ciblée possible, si les données ne sont pas tenues à jour ou ne le sont qu'en partie, l'évaluation qu'on en tire n'a que de peu de valeur. Les données étant relevées en grande partie sur la base de déclarations personnelles, leur qualité dépend fortement de la régularité avec laquelle chaque médecin vérifie et actualise celles qui le concernent. A cet égard, la communication et la motivation jouent un rôle capital. En mars 2009, le projet s'achèvera par la publication de la statistique médicale de 2008, mais la mise à jour périodique des données se poursuivra bien entendu au-delà de cette date.

\section{Coup d'envoi}

Une réunion initiale a eu lieu le 24 mai 2007, rassemblant la direction du projet et dix représentants de diverses organisations*. Ces personnes, de même que plusieurs collaborateurs de la FMH, forment ensemble un groupe d'accompagnement scientifique ainsi que des groupes de travail chargés respectivement des domaines «clients», «politique» et «application technique». L'objectif visé est, d'une part, de procéder à un transfert de connaissances et, d'autre part, de promouvoir l'acceptation de la statistique médicale de la FMH révisée, tant sur le plan interne qu'externe.

Les participants ont abordé, entre autres, les thèmes principaux ci-après et discuté de premières propositions de solutions: 
- l'activité professionnelle du médecin doit être définie en fonction de trois dimensions: lieu, contenu et ampleur. La différence entre médecins indépendants et salariés devra également être repensée lors de la révision;

- la définition des médecins de premier recours et la façon de les recenser doit être revue ou remaniée:

- il convient de recenser le taux d'occupation afin de tenir compte, entre autres, de la part croissante de femmes médecins et de l'augmentation du travail à temps partiel qui va de pair;

- en principe, la statistique médicale de la FMH ne doit pas assumer de tâches liées à la politique de la santé, mais se concentrer sur les aspects purement médicaux. Les variables et les définitions devraient être élaborées sur la base du secteur d'activité essentiel du médecin et la statistique devrait rester centrée sur les personnes.

\section{Conclusion et suite du processus}

De l'avis de la direction du projet, la séance initiale a été utile et réussie. Un des points cru- ciaux a certainement été la confirmation du fait que tous les participants reconnaissent le bienfondé du projet et sont prêts à l'accompagner. Les remarques et les critiques qu'ils ont formulées offrent une base bienvenue pour la suite du processus. Nous tenons à les remercier ici toutes et tous de leur engagement. Depuis lors, le groupe d'accompagnement scientifique s'est réuni pour une première séance de travail. Sa première tâche consiste à choisir les variables et les indicateurs et à élaborer des propositions de définition. Ces paramètres feront l'objet d'une procédure de consultation en temps utile.

La direction du projet tient beaucoup à informer régulièrement les membres de la FMH sur l'évolution de celui-ci. Une information sur le projet de révision de la statistique médicale de la FMH sera donc publiée périodiquement dans ces colonnes.

\section{Référence}

1 Statistique médicale FMH 2006. Bull Méd Suisses. 2007;88(12):508-28. 